\title{
Real-time Defogging of Single Image of loTs-based Surveillance Video Based on MAP
}

\author{
Xin LIU
}

\begin{abstract}
Due to the atmospheric scattering phenomenon in fog weather, the current monitoring video image defogging method cannot estimate the fog density of the image. This paper proposes a real-time defogging algorithm for single images of loTs surveillance video based on maximum a posteriori (MAP). Under the condition of single image sequence, the posterior probability of the high-resolution single image is set to the maximum, which improves the MAP design super-resolution image reconstruction. This paper introduces fuzzy classification to calculate atmospheric light intensity, and obtains a single image of loTs surveillance video by the atmospheric dissipation function. The improved algorithm has the largest signal-to-noise ratio after defogging, and the maximum value is as high as $40.99 \mathrm{~dB}$. The average time for defogging of 7 experimental surveillance video images is only $2.22 \mathrm{~s}$, and the real-time performance is better. It can be concluded that the proposed algorithm has excellent defogging performance and strong applicability.
\end{abstract}

Keywords: Internet of Things (IOT); MAP; monitoring; real-time defogging; single image; video

\section{INTRODUCTION}

At present, TV monitoring systems and intelligent transportation systems are deeply in the form of Internet of Things monitoring. Such systems also have certain drawbacks while they are realizing the intelligence of people's lives. For example, when IoTs monitoring system is operating in weather such as haze, rain, sand and dust storms, the image clarity of the outdoor vision system will be affected, which has a direct impact on the monitoring performance of IoTs monitoring system. The antiinterference performance is poor [1], which seriously affects the quality of service for production and life [3, 4].

Therefore, how to achieve high precision and omnibearing defogging of IoTs surveillance video images is one of the hot issues in the current IoTs surveillance application field. Literature [5] proposed a single-image defogging technique based on dark channels. Atmospheric light is introduced to reconstruct fog-free images and transmission images. Atmospheric light is the brightness of the scene without fog. In this paper, the transmission map based on the adaptive droplet density is selected to reconstruct the best color contrast of the image, and the Laplace filter and the pilot filter are used to refine the transmission map. This method enhances the color contrast of the foggy video image, but it ignores the optimization of the image resolution, and the defogging effect is not ideal. Literature [6] proposed an image-defogging algorithm based on an image degradation model. In order to enhance the image transmission, the global irradiance of the opaque area of the video image has evaluated and monitored to obtain more accurate scene depth information. The implementation process of this method has simple steps, but the single and multiple types of images are confusing, which results in the lack of demisting pertinence and affects the demisting effect. Literature [7] proposed an image-defogging algorithm based on different color wavelength compensation. In order to calculate the red, green and blue (RGB) three-channel transmittance, the monitoring image is processed, and the light attenuation coefficients of different wavelengths are obtained from the dark channel map of the values in the image. In this paper, the modified parameters are introduced into the atmospheric scattering model. Literature [8] proposed a single input image-defogging algorithm based on variational method. In order to obtain the corresponding Euler-Lagrange equation, it is calculated using the negative gradient descent method. The window adaptive method is selected to eliminate the blocking effect, and a good initial value is obtained. This method has obvious defogging effect, but the image detail information is seriously lost after defogging.

In order to solve the problems of the above traditional methods, a MAP-based real-time defogging algorithm for single images of IoTs surveillance video is proposed. This paper adopts MAP to improve the Super-resolution image reconstruction to obtain the foggy concentration point estimation, and iteratively obtains the optimal solution, which effectively improves the information retention rate of the image and shortens the time for defogging. According to the atmospheric dissipation function, a single image of the IoTs surveillance video after demisting is obtained. The experimental results verify that the proposed algorithm can effectively achieve reasonable demisting of single images of IoTs surveillance video.

\section{MATERIAL METHODS}

\subsection{Improvement of Super Resolution Image Reconstruction Algorithm Based on MAP}

The single image of the surveillance video of IoTs will become vague or indistinct in severe weather conditions, and the resolution of the image is extremely low. In order to achieve high-precision defogging of single images of IoTs surveillance video, the posterior probability of highresolution single image is set to the maximum under the condition of single image sequence to obtain highresolution single image of IoTs surveillance video; this process of super resolution reconstruction is estimated for maximum posterior $[9,14]$. Then:

$\widehat{Y}_{M A P}=\arg \max [Q s\{Y \mid X\}]$

Among them, argmax means to obtain the parameter with the maximum score. In Eq. (1), the parameter that makes the posterior probability of the high-resolution single image of the IoTs surveillance video appear as the 
maximum parameter is described. $\hat{Y}_{M A P}$ describes the result of super-resolution image reconstruction after maximum posterior estimation. $Q s\{Y \mid X\}$ represents the maximum likelihood function.

This article uses Bayesian formula to convert $\hat{Y}_{M A P}$ as follows:

$\widehat{Y}_{M A P}=\arg \max \left[\frac{Q s\{X \mid Y\} Q s\{Y\}}{Q s\{X\}}\right]$

Directly this paper removes the denominator that does not interfere with $\hat{Y}_{M A P}$ in the formula. Then:

$$
\widehat{Y}_{M A P}=\arg \max [Q s\{X \mid Y\} Q s\{Y\}]
$$

The logarithm on the right side of the extraction Eq (3) can be obtained:

$$
\widehat{Y}_{M A P}=\arg \max [\log Q s\{X \mid Y\}+\log Q s\{Y\}]
$$

In the formula, the logarithm $Q s\{X \mid Y\}$ of the overall probability function of the high-resolution image of IoTs surveillance video, and the logarithm of the prior probability $Q s\{Y\}$ of the high-resolution image $Y$ of IoTs surveillance video are sequentially set to $\log Q s\{X \mid Y\}$ and $\log Q s\{Y\}$. IoTs surveillance video under severe weather conditions, the existing low-resolution image is set to $X$. The a priori probability of the high-resolution image $Y$ of the surveillance video of IoTs is described as $Q s\{X\}$.

If there is Gaussian noise with a mean value of 0 and a variance of $\beta_{h}^{2}$ in a single image of IoTs surveillance video, the overall probability function that can be estimated for low-resolution image vector $x_{h}$ is as follows.

$$
Q s\left(x_{h} \mid Y\right)=\prod_{\forall y, x}^{Q} \frac{1}{\varepsilon_{h} \sqrt{2 \pi}} \exp \left(-\frac{\left(\hat{x}_{h}-x_{h}\right)^{2}}{2 \varepsilon_{h}^{2}}\right)
$$

Among them, the simulated low-resolution IoTs surveillance video image is set to $\widehat{x}_{h}$. Q represents the probability coefficient. $x_{h}$ represents the low resolution surveillance video image vector. $\varepsilon_{h}$ represents the image local smoothness.

If there is no overlap among individual images of IoTs monitoring video, then the estimation function of the fog concentration point of all low-resolution image sequences is as follows.

$$
Q s(Y)=\exp \left[-\left(\frac{1}{\varphi}\|D Y\|^{2}\right)\right]
$$

In the formula, $\varphi$ represents the control vector of foggy concentration, which can control the peak value of the probability density distribution within a reasonable range. $D$ describes the linear high-pass filter operator, which can realize the operator selection:

$$
\begin{aligned}
& \frac{\partial^{2} g(x, y)}{\partial^{2} y}+\frac{\partial^{2} g(x, y)}{\partial^{2} x}=g(y-1, x-1)+g(y-1, x)+ \\
& +g(y-1, x+1)+g(y, x-1)-8 g(y, x)+g(y, x+1)+ \\
& +g(y+1, x-1)+g(y+1, x)+g(y+1, x+1)
\end{aligned}
$$

Among them, $\partial$ describes the existence of quantifiers means that there are one or several. $g$ represents the constant term coefficient. $y$ and $x$ both represent the vector factors of high and low resolution. Eq. (5) and Eq. (6) are imported into Eq. (4).

$$
Y_{M A P}=\arg \max \left[\log \frac{M}{\varepsilon_{h} \sqrt{2 \pi}}-\sum \frac{\left(\hat{x}_{h}-x_{h}\right)^{2}}{2 \varepsilon_{h}^{2}}-\frac{1}{\varphi}\|D Y\|^{2}\right]
$$

Among them, the example in the brackets on both sides of the equal sign represents the constant term. Then the negative sign of the remaining two items is changed to a positive sign, and the same noise variance is set for each single image, then the above polar problem of maximization becomes the problem of minimization [10]. The minimization problem is as follows.

$Y_{M A P}=\arg \min \left[\sum\left(\hat{x}_{h}-x_{h}\right)^{2}+\frac{2 \varepsilon_{h}^{2}}{\varphi}\|D Y\|^{2}\right]$

Among them, the result of super-resolution image reconstruction after minimization operation is described as $Y_{M A P}$.

Assuming $\frac{2 \varepsilon_{h}^{2}}{\varphi}=b$, then:

$Y_{M A P}=\arg \min \left[\|X-B Y\|^{2}+b\|D Y\|^{2}\right]$

Among them, $b$ represents the regularization parameter. $B$ describes the low-resolution pixel matrix. There must be a necessary condition for minimization in the above formula. To this end, $\|X-B Y\|^{2}+b\|D Y\|^{2}$ sets the partial derivative of $Y$ to 0 .

$$
\begin{aligned}
& 2 B^{\mathrm{T}}(B Y+X)+2 b D^{\mathrm{T}}=0 \\
& \left(B^{\mathrm{T}} B+b D^{\mathrm{T}} \mathrm{P}\right) Y=B^{\mathrm{T}} X
\end{aligned}
$$

Among them, $P$ describes the nonlinear high-pass filter operator. When reconstructing super-resolution images, it is necessary to use iteration to obtain the best solution because of the large amount of calculation [11].

Eq. (10) shows that the regularization parameter $b$ affects the relative contribution value of $\|X-B Y\|^{2}+$ $b\|D Y\|^{2}$ and $\|D Y\|^{2}$ during the solution, and the distortion level and smoothing level effective for reconstruction have an impact [12]. This method adopts the iterative process to update $b$ to obtain the reconstructed image. The newly 
acquired reconstructed image will be used in the subsequent iteration of $b$. The regularization parameter is invalid if it is lower than 0 , so its value must be greater than 0 . If the value $\|X-B Y\|^{2}$ is large the value of $\mathrm{b}$ and $\| X-$ $B Y \|^{2}$ must have a proportional relationship. Because $\mathrm{P}$ represents a high-pass filter operator, $\|D Y\|^{2}$ is larger, and the edge and contour of a single image of the IoTs surveillance video. Therefore, $b$ must be inversely correlated with $\|D Y\|^{2}$. In summary, the method for solving the regularization parameters is as follows.

$$
b_{h+1}=\operatorname{In}\left(\mu * \frac{\left\|X-B Y_{h}\right\|^{2}}{\left\|D Y_{h}\right\|^{2}+s}+1\right)
$$

Among them, the regularization parameter of the $h+$ 1 iteration and the reconstructed image of IoTs surveillance video obtained by iteration are sequentially set to $b_{h+1}$ and $Y_{h} . s$ belongs to a very small number, which can guarantee that the denominator value is not equal to $0 . \mu$ represents the convergence correction factor. When iteratively solving, the algorithm obtains regularization parameters, updates the reconstructed image using the following formula, and sets the number of iterations until the end of the iteration.

$$
Y_{h+1}=Y_{h}+\left[B^{\mathrm{T}} X-\left(B^{\mathrm{T}} B+b_{h+1} D^{\mathrm{T}} D\right) Y_{h}\right]
$$

Among them, $T$ represents the transpose parameter.

The improved algorithm steps are as follows. After obtaining the initial estimation image of the highresolution reconstruction by the surveillance video of IoTs through the convex set projection, it is reconstructed by the MAP method. The detailed process is as follows.

(1) The single low-resolution image is converted into a corresponding high-resolution image by interpolation and enlargement [13].

(2) Perform pre-processing on high-resolution image contours. This pre-processing is to overcome the disadvantages of image contour blur [2].

(3) After obtaining the outline information of a single lowresolution image through the canny edge algorithm, it is interpolated, enlarged, and placed in the corresponding high-resolution image.

(4) Through multiple iterations of the convex set projection method, a set of non-finely reconstructed IoTs surveillance video images can be obtained [15].

(5) This paper adopts MAP algorithm to achieve fine image reconstruction of Internet of Things surveillance video [16].

\subsection{Single Image-Defogging Algorithm}

Drawing on fuzzy set theory, a single image defogging algorithm based on fuzzy set classification is used to achieve defogging of single high-resolution images of IoTs surveillance video [17].

The process of defogging algorithm is as follows.

(1) The fuzzy set principle is used to classify the sky part and the non-sky part in a single high-resolution image of the IoTs surveillance video. The atmospheric light intensity $G$ of the non-sky part is calculated using the dark channel principle [18].
(2) Atmospheric light curtain $U(i, j)$ is estimated and removed by the bilateral filtering method, to realize the single-resolution defogging of the high-resolution images of IoTs surveillance video. The calculation speed of this method is fast, $i$ and $j$ represent pixels.

\subsubsection{Calculation of Atmospheric Light Intensity Based on Fuzzy Classification}

If the atmospheric light intensity $G$ is calculated directly from the highest pixel value, a high amount of noise or white objects will have undesirable interference with its calculation accuracy [19]. To this end, this paper uses the fuzzy set partition mode to divide the single image of IoTs surveillance video into the sky part and the non-sky part, and calculates the atmospheric light intensity $G$ of the non-sky part with high precision through the dark channel. (1) Image area division

The sky part has the properties of high brightness and balanced grayscale. In the IoTs surveillance video image, the average values of the chroma $K$, saturation $R$, and brightness $L$ of the sky part are large, but the standard deviation is not large [20]. Therefore, the sub-picture feature vector $Y_{K R L}$ of a single image of IoTs surveillance video is mainly obtained through the mean and standard deviation of $K, R$, and $L$. Then:

$$
Y_{K R L}=\left(F_{K}, F_{R}, F_{L}, R_{K}, R_{R}, R_{L}\right)^{\mathrm{T}}
$$

Among them, $F_{K}, F_{R}$ and $F_{L}$ represent the average value of $K, R, L . R_{K}, R_{R}$ and $R_{L}$ represents the standard deviation of $K, R, L$.

After obtaining the sub-picture feature vectors of a single image of IoTs surveillance video, all sub-pictures are divided into a sky part and a non-sky part through a fuzzy classification algorithm [21]. The algorithm steps are as follows.

Step 1: the single high-resolution image of IoTs surveillance video is divided into $50 \times 50$ sub-pictures, where the sub-pictures are described as $Q_{1}, Q_{2}, \ldots, Q_{n}$.

Step 2: the feature vector of each subgraph is calculated as follows.

Step 3: we set the iteration end threshold $\theta$ and the maximum number of iterations $\mathrm{Z}$. The equally divided $\mathrm{M}$ samples are set as the initial classification samples, and the clustering matrix is calculated in each cluster of the sky part and the non-sky part of the classification sample $U(0)$.

Step 4: If after step $h-1$ iteration, the fuzzy membership matrix $\mathrm{V}_{(h-1)}$, sky part and non-sky part clustering matrix $\mathrm{U}_{(j-1)}$, of each high-resolution image sample have been obtained, then step $h$ iteration. After that, the fuzzy membership matrix of the single high-resolution image sample of the surveillance video of IoTs is as follows.

$V_{i j}(h)=\left\{\begin{array}{c}\left.1 / \sum_{q=1}^{M} V(h-1) / U(j-1)\right)^{2} \\ 0\end{array}\right.$

Step 5: Import the fuzzy membership matrix $V(h)$ of the sample, then the clustering matrix of each class in the sky part and the non-sky part after iteration in step $h$ is as 
follows.

$$
U_{i}(h)=\left[\sum_{i=1}^{M}\left(V_{i j}(h)\right)^{2} i\right] /\left[\sum_{i=1}^{M}\left(V_{i j}(h)\right)^{2}\right]
$$

The clustering center matrix $U_{i}(h)$ is calculated and a reasonable matrix norm is used to compare $V(h-1)$, with $V(h)$. If $\|V(h)-V(h-1)\|<\theta$, the iteration ends, otherwise set $h=h+1$ and jump to step 4 .

Step 6: Output the classification results of the sky part and non-sky part of the IoTs surveillance video image.

(2) Calculation of atmospheric light intensity

Using the dark channel a priori theory, the atmospheric light intensity $\mathrm{G}$ of the non-sky part of the sub-image is calculated, which can overcome the adverse interference of high-level noise or high-luminance scenes on the atmospheric light intensity calculation [22].

Using the dark channel a priori theory, we can know that the dark channel diagram of a single image of the IoTs surveillance video is as follows.

$$
O(i, j)=\min _{(i, j) \in \varpi(i, j)}\left(\min _{(i, j) \in \varpi(i, j)} O^{e}(i, j)\right)
$$

Among them, the square interval around the pixel point $(i, j)$ is set as $\varpi(i, j) O^{e}(i, j)$ represents the $O(i, j)$ three primary color channels of red, yellow and blue.

In the dark channel diagram of a single image of IoTs monitoring video, the average pixel intensity value of the original image corresponding to the maximum pixel brightness is the atmospheric light intensity value [23].

\subsubsection{Calculation of Atmospheric Dissipation Function}

Atmospheric dissipation function $U(i, j)$ has two constraints:

(1) The value $U(i, j)$ is not less than 0 , then the dissipation function is non-negative.

(2) $U(i, j) \leq \zeta(i)$, then the value of the dissipation function is lower than the lowest value $\zeta(i)$ of the color component of the defogging image. Because of the fog and haze weather, as the distance from the scene to IoTs surveillance video equipment becomes larger, the interference of ambient light on imaging has an increasing trend. The IoTs surveillance video image has a high degree of fog, and the brightness of the image has an increasing trend.

Bilateral filtering $G_{B}(\theta(i))$ belongs to non-local mean filtering mode.

$$
G_{B}(\theta(i))=\frac{\sum_{j \in Y} \sum_{i \in Y}\left(g_{s}(i, j) g_{r}(\zeta(i), \rho) \beta(\rho-\zeta(j)) \zeta(j)\right)}{\sum_{j \in Y} \sum_{i \in Y}\left(g_{s}(i, j) g_{r}(\zeta(i), \rho) \beta(\rho-\zeta(j))\right)}
$$

In the formula, the Gaussian function is set to $g_{s}(i, j)$, which is able to describe the neighboring value of space. $g_{r}$ describes the Gaussian function in the range of values, which can describe the gray similarity value. $\rho$ describes the strength factor.

Considering that there is a certain degree of similarity among the single pixels, in order to set the weight of the filter, the pixels with a smaller distance from the center pixel and a lower grayscale difference are set with a larger weight. Pixels with a smaller distance from the center pixel and a larger grayscale difference will be set with a smaller weight [23]. The detailed calculation process is:

Step 1: Set the dark image $O(i, j)$. If the grayscale image is $\zeta(i, j)$, then:

$O(i, j)=\zeta(i, j)$

and, $O(i, j)=\min _{(i, j) \in \varpi(i, j)} \zeta(i, j)$.

Step 2: Calculate the local mean and local standard deviation of the dark image $O(i, j)$.

Step 3: Calculate the atmospheric dissipation function.

Step 4: If the atmospheric dissipation function meets the constraint $0 \leq U(i, j) \leq O(i, j)$, then set it to:

$U(i, j)=\max (\min (\sigma O(i, j), O(i, j)), 0)$

In the formula, $\sigma$ describes the adjustment coefficient that can improve the image authenticity.

Step 5: the atmospheric light intensity $G$ is used to calculate and optimize the transmission image, then optimize the field depth of the IoTs surveillance video image, and in actual application, even in sunny and foggy weather, the IoTs surveillance video will also be interfered by atmospheric particles. If all the fog is removed, it will reduce the authenticity of the image, so use the adjustment coefficient $\sigma$ to retain a small amount of fog and optimize the image authenticity [24]. Therefore the projection is set to the following relationship.

$k(i, j)=1-\sigma \frac{U(i, j)}{G}$

Step 6: In order to avoid $k(i, j)$ that the value of 0 is too close to 0 , which sets the lower limit $k_{0}$, so the single image of the IoTs surveillance video after defogging is:

$$
\Phi(i, j)=\frac{\zeta(i, j)-G}{\max \left(k(i, j), k_{0}\right)}+G
$$

\section{RESULTS}

In order to test the effectiveness of the algorithm of this paper on the single image defogging of IoTs monitoring video, a simulation experiment environment is established by MATLAB R2019 software in Windows 10 system. In the standard test image of the SIPI image database, select the IoTs surveillance video image of a certain traffic trunk. The IoTs surveillance video image is used for security in a community. In addition, the two IoTs surveillance video images have fogging phenomenon. We can see Fig. 1 and Fig. 2 for details. The single image of IoTs surveillance video processed by the algorithm in this paper is shown in Fig. 3 and Fig. 4. 


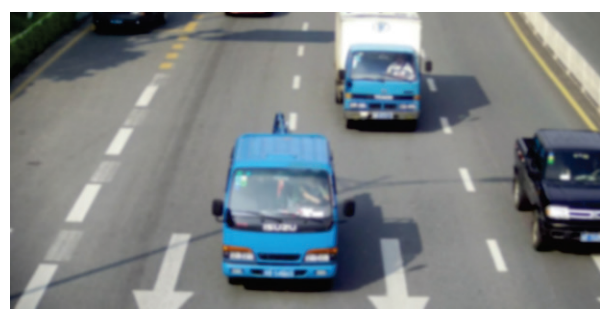

Figure 1 Video image of Internet of things monitoring of a traffic trunk Line

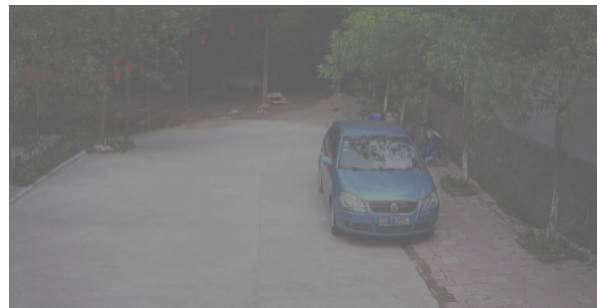

Figure 2 Video image of Internet of things monitoring used for security in a community

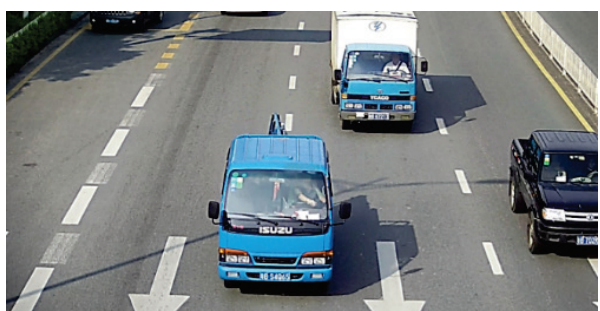

Figure 3 The algorithm in this paper processes the video image of post traffic monitoring

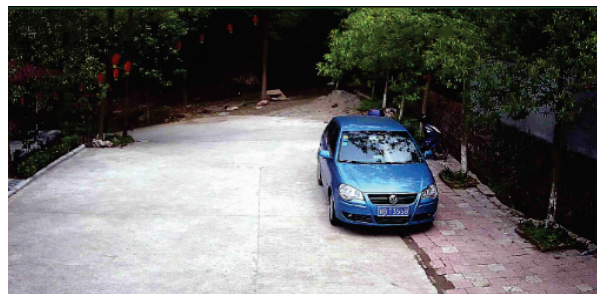

Figure 4 Post security monitoring image processed by the algorithm in this paper
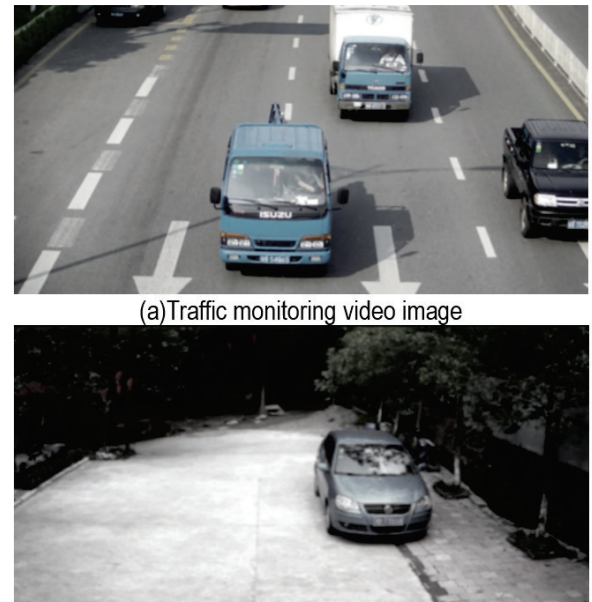

(b)Security monitoring image

Figure 5 Defogging effect of adaptive image defogging algorithm based on mean unequal relation optimization

It can be seen intuitively from Fig. 3 and Fig. 4 that after the algorithm processing in this paper, the clarity of the two surveillance video images of IoTs can be improved and the effect of defogging is better. In order to highlight the advantages of the defogging performance of the algorithm in this paper, an adaptive image defogging algorithm based on the optimization of the mean inequality relationship, an image defogging algorithm combining convolutional neural network and dynamic ambient light is also used to defog Fig. 1 and Fig. 2. The defogging effect diagram is shown in Fig. 5 and Fig. 6.

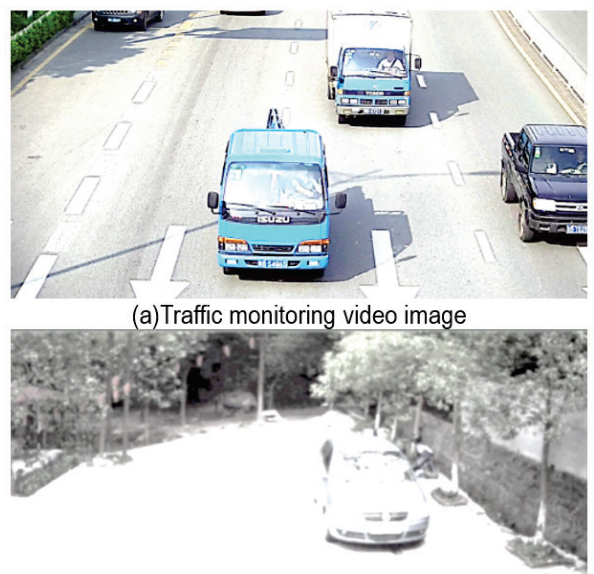

(b)Security monitoring image

Figure 6 Image defogging effect combined with convolution neural network and dynamic ambient light

It can be seen from Fig. 5 and Fig. 6 that the adaptive image defogging algorithm optimized based on the inequality of the mean value, the image defogging algorithm combined with the convolutional neural network and the dynamic ambient light have poor defogging effect on the traffic monitoring video images and security monitoring images. Compared with the algorithm in this paper, the gap is significant. The mean value unequal relationship optimization-defogging algorithm teaches it to monitor the video image after defogging, the image color is distorted, and the car license number in the two images is blurred. After defogging combined with convolutional neural network and dynamic ambient light, the image exposure is higher [25]. In contrast, the algorithm in this paper has the best defogging effect.

The above test results are all based on visual analysis of the defogging effect of the algorithm of this article on IoTs monitoring video image. In order to accurately analyze the use effect of the algorithm in this paper, from the perspective of numerical verification, the three signals are tested by the peak signal-to-noise ratio and structural similarity index to test the defogging effect of the IoTs surveillance video image. IoTs monitoring video images are divided into traffic monitoring images, security monitoring images, agricultural monitoring images, home monitoring images, fire-monitoring images, hospital monitoring images, campus-monitoring images. The peak signal-to-noise ratio and structural similarity index of several IoTs surveillance video images after the three algorithms are defogged and are shown in Tab. 1 and Tab. 2.

It can be seen from the analysis of Tab. 1 and Tab. 2 that this paper adopts three algorithms to defog the 7 kinds of Internet of Things surveillance video images, and the image signal-to-noise ratio of the image after defogging is the largest, and the maximum value is as high as $40.99 \mathrm{~dB}$.

The other two algorithms have similar image structure 
index after defogging. It can be proved that the algorithm of this paper has the best effect on defogging different types of Internet of Things surveillance video images.

This paper tests the time complexity of three algorithms for demisting 7 kinds of IoTs surveillance videos. 7 Internet of things monitoring includes traffic monitoring images, security monitoring images, agricultural monitoring images, home monitoring images, fire monitoring images, hospital monitoring images, campus monitoring images. The test results are shown in Tab. 3. The greater the signal-to-noise ratio, the less distortion the image has after defogging. The signal-tonoise ratio of the other two algorithms after defogging is lower than $40.00 \mathrm{~dB}$. The similarity index of the image structure after defogging in this algorithm is closest to 1 . Then there is no loss in the image structure of the algorithm after defogging.

Table 1 Comparison results of peak signal-to-noise ratio of monitoring video images after demisting with three algorithms/ unit DB

\begin{tabular}{|c|c|c|c|}
\hline $\begin{array}{c}\text { Types of Internet of things monitoring } \\
\text { video images }\end{array}$ & $\begin{array}{c}\text { Algorithm in this } \\
\text { paper }\end{array}$ & $\begin{array}{c}\text { Adaptive image defogging algorithm based } \\
\text { on mean unequal relation optimization }\end{array}$ & $\begin{array}{c}\text { Image defogging algorithm based on } \\
\text { convolution neural network and dynamic } \\
\text { ambient light }\end{array}$ \\
\hline Traffic monitoring image & 40.56 & 35.33 & 37.11 \\
\hline Security monitoring image & 40.33 & 35.22 & 37.09 \\
\hline Agricultural monitoring image & 40.56 & 35.33 & 37.45 \\
\hline Home monitoring image & 40.34 & 35.33 & 37.45 \\
\hline Fire monitoring image & 40.32 & 35.59 & 37.43 \\
\hline Hospital monitoring image & 40.33 & 35.23 & \\
\hline
\end{tabular}

Table 2 Comparison results of structure similarity index of monitoring video images after demisting by three algorithms/ unit DB

\begin{tabular}{|c|c|c|c|}
\hline $\begin{array}{c}\text { Types of Internet of things monitoring } \\
\text { video images }\end{array}$ & $\begin{array}{c}\text { Algorithm in this } \\
\text { paper }\end{array}$ & $\begin{array}{c}\text { Adaptive image defogging algorithm based } \\
\text { on mean unequal relation optimization }\end{array}$ & $\begin{array}{c}\text { Image defogging algorithm based on } \\
\text { convolution neural network and dynamic } \\
\text { ambient light }\end{array}$ \\
\hline Traffic monitoring image & 0.9987 & 0.9123 & 0.8823 \\
\hline Security monitoring image & 0.9978 & 0.9212 & 0.8799 \\
\hline Agricultural monitoring image & 0.9978 & 0.9188 & 0.8789 \\
\hline Home monitoring image & 0.9987 & 0.9123 & 0.8788 \\
\hline Fire monitoring image & 0.9978 & 0.9212 & 0.8789 \\
\hline Hospital monitoring image & 0.9978 & 0.9188 & 0.8788 \\
\hline Campus monitoring image & 0.9983 & 37.56 & \\
\hline
\end{tabular}

Table 3 Comparison results of time complexity of three algorithms / unit s

\begin{tabular}{|c|c|c|c|}
\hline $\begin{array}{c}\text { Types of Internet of things monitoring } \\
\text { video images }\end{array}$ & $\begin{array}{c}\text { Algorithm in this } \\
\text { paper }\end{array}$ & $\begin{array}{c}\text { Adaptive image defogging algorithm based } \\
\text { on mean unequal relation optimization }\end{array}$ & $\begin{array}{c}\text { Image defogging algorithm based on } \\
\text { convolution neural network and dynamic } \\
\text { ambient light }\end{array}$ \\
\hline Traffic monitoring image & 2.34 & 6.56 & 9.43 \\
\hline Security monitoring image & 2.22 & 6.68 & 9.56 \\
\hline Agricultural monitoring image & 2.19 & 6.76 & 9.66 \\
\hline Home monitoring image & 2.22 & 6.56 & 9.56 \\
\hline Fire monitoring image & 2.19 & 6.68 & 9.43 \\
\hline Hospital monitoring image & 2.19 & 6.68 & 9.56 \\
\hline Campus monitoring image & 2.21 & 6.64 & 9.6 \\
\hline
\end{tabular}

Table 4 Comparison results of effective detail intensity of image after demisting by three algorithms

\begin{tabular}{|c|c|c|c|c|c|c|c|c|c|}
\hline \multirow{2}{*}{$\begin{array}{l}\text { IOTs monitoring video } \\
\text { type }\end{array}$} & \multicolumn{3}{|c|}{ Algorithm in this paper } & \multicolumn{3}{|c|}{$\begin{array}{c}\text { Adaptive image defogging algorithm } \\
\text { based on mean unequal relation } \\
\text { optimization }\end{array}$} & \multicolumn{3}{|c|}{$\begin{array}{c}\text { Image defogging algorithm based on } \\
\text { convolution neural network and dynamic } \\
\text { ambient light }\end{array}$} \\
\hline & $\begin{array}{l}\text { Haze } \\
\text { pollution } \\
\text { level } 1\end{array}$ & $\begin{array}{l}\text { Haze } \\
\text { pollution } \\
\text { level } 2\end{array}$ & $\begin{array}{l}\text { Haze } \\
\text { pollution } \\
\text { level } 3\end{array}$ & $\begin{array}{l}\text { Haze } \\
\text { pollution } \\
\text { level } 1\end{array}$ & $\begin{array}{l}\text { Haze } \\
\text { pollution } \\
\text { level } 2\end{array}$ & $\begin{array}{l}\text { Haze } \\
\text { pollution } \\
\text { level } 3\end{array}$ & $\begin{array}{l}\text { Haze } \\
\text { pollution } \\
\text { level } 1\end{array}$ & $\begin{array}{l}\text { Haze } \\
\text { pollution } \\
\text { level } 2\end{array}$ & $\begin{array}{l}\text { Haze } \\
\text { pollution } \\
\text { level } 3\end{array}$ \\
\hline Traffic monitoring image & 0.3432 & 0.4024 & 0.3535 & 0.2923 & 0.3013 & 0.2023 & 0.3123 & 0.3234 & 0.1923 \\
\hline $\begin{array}{c}\text { Security monitoring } \\
\text { image }\end{array}$ & 0.3643 & 0.4024 & 0.3566 & 0.2999 & 0.3022 & 0.2034 & 0.3022 & 0.3233 & 0.1924 \\
\hline $\begin{array}{c}\text { Campus monitoring } \\
\text { image }\end{array}$ & 0.3746 & 0.4011 & 0.3589 & 0.2983 & 0.3232 & 0.2932 & 0.3024 & 0.3234 & 0.1922 \\
\hline $\begin{array}{l}\text { Agricultural monitoring } \\
\text { image }\end{array}$ & 0.3746 & 0.4011 & 0.3567 & 0.2901 & 0.3092 & 0.2834 & 0.3024 & 0.3235 & 0.1902 \\
\hline Fire monitoring image & 0.3777 & 0.4013 & 0.3564 & 0.2912 & 0.3213 & 0.2374 & 0.3033 & 0.3233 & 0.1924 \\
\hline
\end{tabular}

The results in the table show that the time-consuming average value of the algorithm in this paper for de-fogging 7 kinds of Internet of Things surveillance video images is only $2.22 \mathrm{~s}$, and the average time-consuming value of the two comparison algorithms is $4.42 \mathrm{~s}$ and $7.33 \mathrm{~s}$ more than the algorithm in this paper. In this paper, the algorithm has the lowest time complexity and the highest defogging efficiency.

The three algorithms are used in order to defrost the traffic monitoring images, security monitoring images, campus monitoring images, agricultural monitoring images, and fire monitoring images (outdoor) in the environment where the haze concentration level is 1,2 , and 3 . Test the effective detail intensity of the image after the three algorithms defog. The test results are shown in Tab. 4.

The test results in the table show that the algorithm of this paper has the lowest effective detail intensity of the 
image after defogging traffic monitoring images, security monitoring images, campus monitoring images, agricultural monitoring images, fire monitoring images (outdoors) of different smog concentration pollution levels. The values are $0.3432,0.4011$, and 0.3535 in turn. The other two algorithms defog several IoTs surveillance video images under different levels of smog concentration pollution. The maximum effective detail intensity of the image is lower than the minimum value of the algorithm in this paper. It is verified from this that the algorithm of this paper improves the anti-interference performance of the defogging work, and the use limit is extremely small.

\section{DISCUSSION}

Combined with the research content of this article, the discussion on the needs of IoTs for the development of monitoring systems is implemented. This article believes that if the camera is imagined as an adult's eye, then IoTs monitoring device is the human brain, and video monitoring is an indispensable step in IoTs perception $\mathrm{s}$ eyes. However, there are many data in IoTs monitoring equipment. If the monitoring data processing is implemented only in a manual manner, there is a drawback of low working efficiency. Therefore, at this moment, it is necessary to develop an intelligent and real-time defogging algorithm for real-time IoTs surveillance video images to improve the quality of IoTs surveillance video images. This paper proposes a MAP-based real-time defogging algorithm for IoTs surveillance video single images, which meets this need and can be used as an effective algorithm for IoTs surveillance video single image governance optimization.

\section{CONCLUSION}

This paper focuses on the issue of optimizing the single image quality of IoTs surveillance video, proposes a MAPbased real-time defogging algorithm for IoTs surveillance video single image, and verifies its effectiveness through experiments. The detailed verification results are as follows:

(1) From a visual point of view, after the algorithm in this paper is processed, the clarity of the IoTs surveillance video image of a certain traffic trunk line and the IoTs surveillance video of a certain community for security can be improved. Its advantage is that the defogging effect is better.

(2) After three algorithms to defog 7 experimental IoTs surveillance video images, the algorithm has the largest signal-to-noise ratio after defogging, and the maximum value is as high as $40.99 \mathrm{~dB}$. The similarity index of the image structure after defogging in this paper is closest to 1 . Its advantage is that there is no loss in the image structure after defogging in this algorithm.

(3) The time-consuming average value of the algorithm for the de-fogging of 7 experimental IoTs monitoring video images is only $2.22 \mathrm{~s}$. Its advantage has the lowest time complexity and can realize real-time de-fog.

(4) The algorithm of this paper under different levels of smog concentration pollution, after removing fog from traffic monitoring images, security monitoring images, campus monitoring images, agricultural monitoring images, fire monitoring images (outdoors), the minimum value of image effective detail intensity is $0.3432,0.4011$, 0.3535 . The application in this paper not only has a positive effect on the image defogging work, but also improves the anti-interference performance of the defogging work, and its application is extremely limited.

\section{Acknowledgment}

The work was supported by the Fundamental Research Funds for the Central Universities (Nos. XDJK2018C084).

\section{REFERENCES}

[1] Jiang, Y., Sun, C., \& Zhao, Y. (2017). Fog Density Estimation and Image Defogging Based on Surrogate Modeling for Optical Depth. IEEE Transactions on Image Processing, 26(7), 3397-3409. https://doi.org/10.1109/TIP.2017.2700720

[2] Zhang, W., Dong, L., \& Pan, X. (2019). Single Image Defogging Based on Multi-Channel Convolutional MSRCR. IEEE Access, 7(1), 72492-72504. https://doi.org/10.1109/ACCESS.2019.2920403

[3] Hu, H. M., Guo, Q., \& Zheng, J. (2019). Single Image Defogging Based on Illumination Decomposition for Visual Maritime Surveillance. IEEE Transactions on Image Processing, 28(6), 2882-2897. https://doi.org/10.1109/TIP.2019.2891901

[4] Li, Y. (2019). The Application of Internet of Things Technology in Environmental Protection. Journal of China Academy of Electronics and Information Technology, (12), 1249-1252.

[5] Tufail, Z., Khurshid, K., \& Salman, A. (2019). Optimization of Transmission Map for Improved Image Defogging. IET Image Processing, 13(7), 1161-1169. https://doi.org/10.1049/iet-ipr.2018.6485

[6] Wang, Y. \& Yuan, P. (2017). Image dehazing based on partitioning reconstruction and entropy-based alternating fast-weighted guided filters. Optical engineering, 56(5), 053111.1-053111.8. https://doi.org/10.1117/1.OE.56.5.053111

[7] Chen, Z., Ou, B., \& Tian, Q. (2019). An improved dark channel prior image defogging algorithm based on wavelength compensation. Earth ence Informatics, 12(4), 501-512. https://doi.org/10.1007/s12145-019-00395-y

[8] Wang, Z., Pan, Z. K., \& Wang, G. D. (2019). A Variational Model for Single Image Dehazing and Denoising. Computer Simulation, 36(02), 181-187.

[9] Cai, C. C., Shen, Y. X., \& Zhao, W. H. (2019). Bridgeless LED Driver Based on Cuk Converter. Journal of Power Supply, 017(004), 57-63.

[10] Jiang, G. H., Wan, H. B., \& Luo, W. C. (2019). Research on internet of things equipment for fluid detection and online monitoring of tobacco leaf quality. Automation \& Instrumentation, (11), 153-156+160.

[11] Luo, X., Mcleod, A. J., \& Pautler, S. E. (2017). Vision-Based Surgical Field Defogging. IEEE Transactions on Medical Imaging, 36(10), 2021-2030. https://doi.org/10.1109/TMl.2017.2701861

[12] Cheng, Z. X. \& Qiao, M. (2019). Optimal Communication Node Selection Algorithm for Intrusion of Internet of Things. Journal of Jilin University (Science Edition), 057(004), 903909.

[13] Oh, S., Lee, J., \& Park, S. (2017). Energy Efficient and Accurate Monitoring of Large-Scale Diffusive Objects in Internet of Things. IEEE communications letters, 21(3), 612615. https://doi.org/10.1109/LCOMM.2016.2634526

[14] Zheng, L. N. (2017). Intelligent monitoring system of power based on Internet of Things. Chinese Journal of Power Sources, 041(003), 493-494. 
[15] Gao, Z., Yang, M., \& Xie, C. (2017). Space target image fusion method based on image clarity criterion. Optical engineering, 56(5), 053102.1-053102.13. https://doi.org/10.1117/1.0E.56.5.053102

[16] Priyadumkol, J., Kittichaikarn, C., \& Thainimit, S. (2017) Crack detection on unwashed eggs using image processing. Journal of food engineering, 209(SEP.), 76-82. https://doi.org/10.1016/j.jfoodeng.2017.04.015

[17] Zhang, R., Cheng, S., \& Guo, C. (2018). Detection Method for Pulverized Coal Injection and Particles in the Tuyere Raceway Using Image Processing. Isij International, 58(2), 244-252. https://doi.org/10.2355/isijinternational.ISIJINT-2017-433

[18] Temel, D. \& Alregib, G. (2018). Traffic Signs in the Wild: Highlights from the IEEE Video and Image Processing Cup 2017 Student Competition [SP Competitions]. IEEE Signal Processing Magazine, 35(2), 154-161. https://doi.org/10.1109/MSP.2017.2783449

[19] Ragan, K. J., Adams, A., \& Sharlet, D. (2018). Halide: Decoupling Algorithms from Schedules for HighPerformance Image Processing. Communications of the ACM, 61(1), 106-115. https://doi.org/10.1145/3150211

[20] Hashimoto, T. \& Murai, S. (2017). Traffic flow measurement by video image processing. Journal of the American college of cardiology, 59(12), 1045-57. https://doi.org/10.1016/j.jacc.2011.09.077

[21] Dang, H. P. \& Chainais, P. (2017). Indian Buffet Process dictionary learning: Algorithms and applications to image processing. International Journal of Approximate Reasoning, 83(Complete), 1-20. https://doi.org/10.1016/j.jjar.2016.12.010

[22] Staehly, S., Friedrich, H., \& Detert, M. (2017). Size Ratio of Fluvial Grains' Intermediate Axes Assessed by Image Processing and Square-Hole Sieving. Journal of Hydraulic Engineering, 143(6), 06017005.1-06017005.6. https://doi.org/10.1061/(ASCE)HY.1943-7900.0001286

[23] Soorya, M., Issac, A., \& Dutta, M. K. (2018). An automated and robust image processing algorithm for glaucoma diagnosis from fundus images using novel blood vessel tracking and bend point detection. International journal of medical informatics, 110 (FEB), 52-70. https://doi.org/10.1088/1475-7516/2017/10/015

[24] Tseng, E., Yu, F., \& Yang, Y. (2019). Hyperparameter optimization in black-box image processing using differentiable proxies. ACM Transactions on Graphics, 38(4), 1-14. https://doi.org/10.1145/3306346.3322996

[25] Hanwell, M. D., Harris, C. J., \& Genova, A. (2019). Tomviz: Open Source Platform Connecting Image Processing Pipelines to GPU Accelerated 3D Visualization. Microscopy and Microanalysis, 25(S2), 408-409.

https://doi.org/10.1017/S1431927619002770

\section{Contact information:}

\section{Xin LIU}

Information Center, Southwest University, No. 2 Tiansheng Road, Beibei District,

Chongqing, 400715, P. R. China

Email: liuxin_swu@163.com 